\title{
HI-selected Galaxies As a Probe of Quasar Absorption Systems
}

\author{
K. Okoshi ${ }^{1}$, M. Nagashima ${ }^{2}$, N. Gouda ${ }^{3}$ and Y. Minowa ${ }^{3}$ \\ ${ }^{1}$ Tokyo University of Science, Oshamanbe, Hokkaido, 049-3514, Japan \\ ${ }^{2}$ Nagasaki University, Japan, ${ }^{3}$ National Astronomical Observatory, Japan
}

\begin{abstract}
We investigate the properties of local HI-selected galaxies detected in blind radio surveys by using semi-analytic galaxy formation models in the hierarchical structure formation scenario. By drawing a detailed comparison between the properties of the HI-selected galaxies and the quasar absorption systems, we investigate a link between the local galaxy population and the quasar absorption systems, particularly for Damped Lyman $\alpha$ absorption (DLA) systems $\left(N_{\mathrm{HI}} \geqslant 10^{20.3} \mathrm{~cm}^{-2}\right)$ and sub-DLA systems $\left(10^{19}<N_{\mathrm{HI}}<10^{20.3} \mathrm{~cm}^{-2}\right)$.

We find that DLA galaxies, in which the HI column densities are as high as those of DLA systems, contribute primarily to the population of local HI-selected galaxies at $M_{\mathrm{HI}} \geqslant 10^{8} M_{\odot}$. By contrast, in the low-mass range $M_{\mathrm{HI}} \leqslant 10^{7} M_{\odot}$, sub-DLA galaxies replace DLA galaxies as the dominant population. The HI-selected galaxies would be a strong probe of DLA/sub-DLA systems.
\end{abstract}

Keywords. galaxies: evolution, quasars: absorption lines, radio lines: galaxies.

\section{Introduction}

The quasar/GRB absorption systems provide us with a unique probe of galaxy formation and evolution processes. In particular, the HI absorption systems have been investigated extensively to place stringent constraint on the physical condition of the galactic gas. However, the origin of the absorption systems are still unclear because the optical counterparts are quite few even at low redshift $z<1$.

Recent blind radio surveys (e.g., the HI Parkes All Sky Survey; HIPASS and the Arecibo Legacy Fast ALFA survey; ALFALFA) have offered large samples of HI-selected galaxies in which the column densities are comparable to those in DLAs. Focusing on the large sample with highly statistical confidence, the local HI-selected galaxies would place constraint on the origin of absorption systems. Here, we investigate a link between the HI emission galaxies and the HI absorption systems.

\section{HI-selected Galaxies \& DLA/sub-DLA systems}

We employ a semi-analytic model for galaxy formation (e.g., Okoshi et al. 2004; Okoshi \& Nagashima 2005)which incorporates basic processes of galaxy formation (e.g., merging process and $\mathrm{SN}$ feedback)in the standard $\Lambda$ CDM universe.

In Figure 1(left panel), we show the HI mass functions (HIMF) of the local galaxy population for our models where the star formation rates (SFR) are high (the HS model) and low (the LS model) (for details, Okoshi et al. 2009). Both results are consistent with the HI observations because the predicted number densities match within the range of the observational data. For the LS model, the high number densities at low-mass end is caused by the SFRs lower than those for the HS model.

In Figure 1(right panel), we present number fractions of $D L A$ galaxies as a function of HI mass $M_{\mathrm{HI}}$. The result for the HS model (solid line) shows that the number fractions 

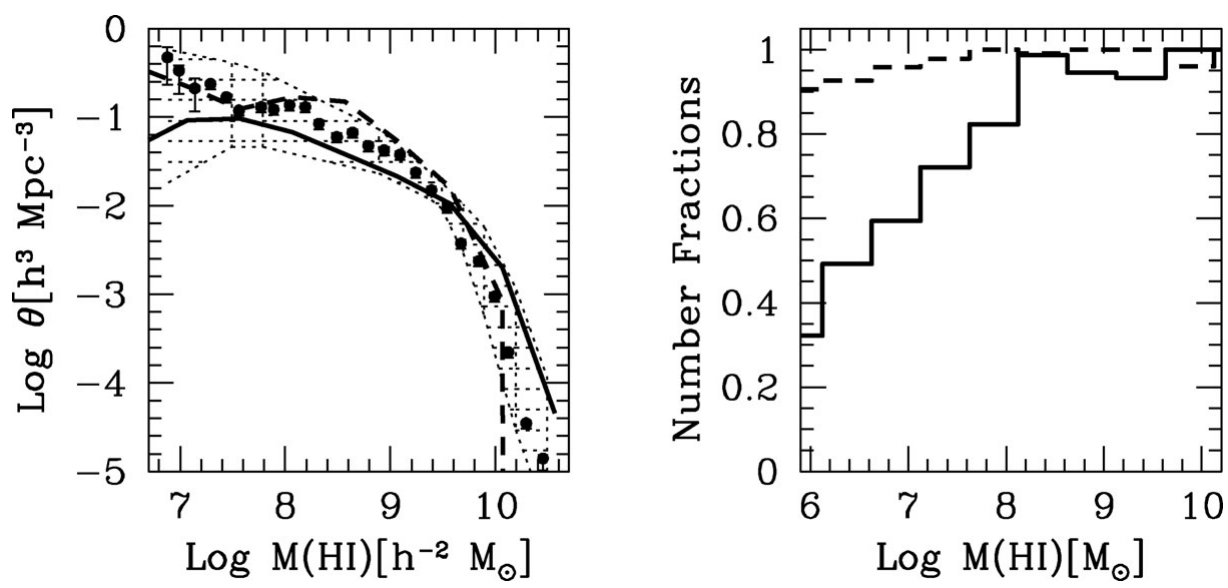

Figure 1. Left panel: HI mass functions of galaxies for the HS (solid line) and the LS (dashed line) models. The points are the observational data provided by the HIPASS (filled circles; Zwaan et al. 2005). The shaded region represents the region of HIMFs given by various blind surveys. Right panel: The histogram of number fractions of DLA galaxies for the HS (solid line) and the LS (dashed line) models, respectively. The number fraction is defined as a ratio of the number densities of DLA galaxies to those of galaxies per bin of HI mass.

are almost unity at $M_{\mathrm{HI}}>10^{8} M_{\odot}$, which suggests a trend that gaseous disks of massive galaxies have HI column densities as high as those in DLA systems.

Noticeably, in the low-mass range $M_{\mathrm{HI}}<10^{8} M_{\odot}$, for the HS model, the number fractions begin to decrease toward the low-mass end, e.g., $\sim 0.7$ at $10^{7.5} M_{\odot}$ and $\sim 0.6$ at $10^{7} M_{\odot}$. This indicates that a small amount of $\mathrm{HI}$ gas of the less massive systems does not produce high enough HI column densities to detect as DLA systems. At the low-mass end, another population, sub-DLA systems, becomes dominant instead of DLA galaxies. The number fractions of sub-DLA galaxies relative to galaxies attain $60-40 \%$ at $M_{\mathrm{HI}}=10^{8} M_{\odot}$ and $80-30 \%$ at $M_{\mathrm{HI}}=10^{7} M_{\odot}$ for the HS and the LS models. If the HI-mass detection limits in blind surveys are low enough to detect less massive systems $\left(M_{\mathrm{HI}} \sim 10^{7} M_{\odot}\right)$, our results suggest that the population detected by the surveys would switch to being dominated by sub-DLA galaxies instead of DLA galaxies. The HI-selected galaxies and the optical counterparts would be a strong probe of DLA/sub-DLA systems that place stringent constraint on key processes of galaxy formation and evolution.

\section{References}

Okoshi, K., Nagashima, M., Gouda, N., \& Yoshioka, S. 2004, ApJ, 603, 12

Okoshi, K. \& Nagashima, M. 2005, ApJ, 623, 99

Okoshi, K., Nagashima, M., Gouda, N., \& Minowa, Y. 2009, submitted to ApJ

Zwaan, M. A., Meyer, M. J., Staveley-Smith, L., \& Webster, R. L. 2005, MNRAS, 359, L30 